\title{
Editorial on "Simultaneous resections of colorectal cancer and synchronous liver metastases: a multi-institutional analysis"
}

\author{
Charlotte Ariyan, MD, Douglas Wong, MD, and Yuman Fong, MD \\ Department of Surgery, Memorial Sloan-Kettering Cancer Center, 1275 York Avenue, New York, New York 10021, USA
}

Liver resection has become the standard treatment for metastatic colorectal cancer. ${ }^{1}$ Such procedures are now justified by the increasing safety of hepatic resections and by documentation of long-term disease-free survival. ${ }^{1,2}$ Metastatic disease confined to the liver is now routinely resected as part of a potentially curative strategy. When patients present with liver metastases synchronous with a colorectal primary, however, the timing of surgical treatment remains controversial. In studies published as late as the 1990s, prohibitive perioperative mortality was reported when combining major liver resections with resection of the colorectal primary. An operative mortality as high as $17 \%$ was reported for such simultaneous resections. ${ }^{3}$ Because of this and other reports, many centers have been reluctant to perform simultaneous resections, but rather have favored resecting the primary and liver metastases at two separate operations. The report in the current issue by Reddy and his colleagues presents the experience of simultaneous resections from three centers over a 20 -year period ${ }^{4}$; these results add to an emerging literature suggesting simultaneous resections to a safe surgical strategy.

The data from the 135 patients analyzed clearly demonstrate that combining minor liver resection with resection of colorectal primary shortened total

Received May 25, 2007; accepted May 27, 2007; published online: September 25, 2007.

Address correspondence and reprint requests to: Yuman Fong, MD; E-mail: fongy@mskcc.org

Published by Springer Science+Business Media, LLC @ 2007 The Society of Surgical Oncology, Inc. hospitalization without compromising safety. However, the authors stopped short of endorsing combined major liver resection and colorectal resection even though such combined resections were also associated with a shorter hospitalization: 9 days compared with 14 days for staged surgery. ${ }^{4}$ This is partly because of the limited number of patients with complete data for both liver resection and colorectal resection. In the 36 simultaneous major liver and colorectal resections, there was an insignificant trend toward a higher operative mortality.

More favorable and definitive outcomes have previously been reported. In the largest single institutional experience to date, Martin and colleagues compared 134 patients subjected to simultaneous resection with 106 patients treated by staged resection. Mortality and complications were equivalent, although total hospital stay and blood loss were superior in the simultaneous resection group. ${ }^{5}$ Even when patients undergoing major liver resections were considered, the outcomes of the 45 patients who had simultaneous resections were superior to those subjected to staged resections. In well-selected patients, in experienced hands, simultaneous liver and colorectal resections can be performed to achieve favorable outcomes.

What is not well described in these reports are a number of the technical considerations that have made simultaneous resections feasible and safe. ${ }^{6}$ During the conduct of simultaneous resections, many experienced liver surgeons will perform the hepatectomy prior to the resection of the primary tumor. The main reason for this is that hepatectomies are generally performed under low central venous pressure 
anesthesia, which decreases blood loss and improves outcome. Performing the liver resection first allows fluid resuscitation during the colorectal resection phase of the combined procedure, effectively limiting the period of relative hypovolemia and low splanchnic blood flow. In addition, performing the hepatectomy first also prevents venous congestion on a fresh anastomosis during the Pringle maneuver. Performing the liver resection first has practical benefits of allowing for a single setup of surgical instruments. Most operative nursing units will allow the instruments used in the liver resection to be used in the colorectal resection, but not allow the instruments used in the "dirty" colorectal resection to be used in the "clean" hepatectomy.

The choice of incisions has also ceased to be an obstacle to simultaneous resections. Although in previous years many surgeons were inclined to believe that major liver resections had to be performed through a subcostal incision, it has become clear that even the most extensive resections can be performed safely through a long midline incision extended from xiphoid to symphysis. The most extensive of hepatectomies can be combined even with low rectal resections and pouch reconstructions, if the patient is a suitable medical and technical candidate.

One question that remains unanswered is whether simultaneous resection provides an equivalent longterm cancer outcome. In a pioneering publication on liver resection for colorectal metastases, Scheele had found that patients subjected to simultaneous resections had a trend toward less favorable long-term survival. $^{2}$ Whether this is due to patient staging, selection, or other factors is unclear. It is possible that patients with smaller tumors were selected for simultaneous resection, as observed in the study of Reddy et al., ${ }^{4}$ and were those more likely to have additional smaller lesions not detectable until later? Whether current effective adjuvant chemotherapies or current cross sectional imaging not available to Scheele's patients may have negated the unfavorable outcome is unknown. Future studies will need to compare the long-term outcomes of the patients subjected to simultaneous or staged resections.

There is no longer doubt that minor liver resections can be performed safely at the same setting as resection of a colorectal primary. However, patients should be selected carefully for combined major liver resections and colorectal resection. The patient's medical condition, extent of primary and metastatic disease, body habitus, as well as the comfort level of the operating surgeon should all influence the choice of sequence of therapy.

\section{REFERENCES}

1. Fong Y, Fortner J, Sun RL, Brennan MF, Blumgart LH. Clinical score for predicting recurrence after hepatic resection for metastatic colorectal cancer: analysis of 1001 consecutive cases. Ann Surg 1999; 230(3):309-18.

2. Scheele J, Stangl R, Altendorf-Hofmann A, Gall FP. Indicators of prognosis after hepatic resection for colorectal secondaries. Surgery 1991; 110(1):13-29.

3. Bolton JS, Fuhrman GM. Survival after resection of multiple bilobar hepatic metastases from colorectal carcinoma. Ann Surg 2000; 231(5):743-51.

4. Reddy S, Pawlik T, Zorzi D, Gleisner A, Ribero D, Assumpcao L, Barbas A, Abdalla E, Choti M, Vauthey JN, Ludwig K, Mantyh C, Morse M, Clary B. Simultaneous resections of colorectal cancer and synchronous liver metastases: a multiinstitutional analysis. Ann Surg Oncol 2007; doi:10.1245/ s10434-007-9522-5.

5. Martin R, Paty P, Fong Y, Grace A, Cohen A, DeMatteo R, Jarnagin W, Blumgart L. Simultaneous liver and colorectal resections are safe for synchronous colorectal liver metastasis. J Am Coll Surg 2003; 197(2):233-41.

6. Cho C, Park J, Fong Y. Liver resection for cancer. ACS Surgery: Principles and Practice 2007; (in press). 